RESEARCH ARTICLE

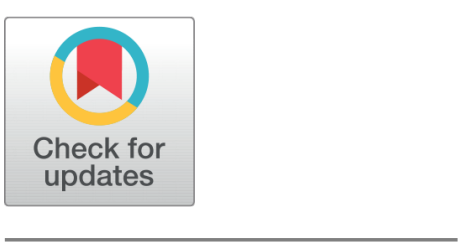

G open access

Received: 22-05-2014

Accepted: 26-07-2015

Published: 09-07-2020

Editor: Dr. Natarajan Gajendran

Citation: Naghshband S, Araghi MAF (2020) Solving the cubic complex Ginzburg-Laundau equation by Homotopy analysis method. Indian Journal of Science and Technology 13(24): 2387-2403. https://doi.org/ 10.17485/IJST/v13i24.54557.90477

*Corresponding author. S Naghshband

Department of Mathematics, Central Tehran Branch, Islamic Azad University, Tehran, Iran s_naghshband@yahoo.com

Funding: None

Competing Interests: None

Copyright: (c) 2020 Naghshband, Araghi. This is an open access article distributed under the terms of the Creative Commons Attribution License, which permits unrestricted use, distribution, and reproduction in any medium, provided the original author and source are credited.

Published By Indian Society for Education and Environment (iSee)

\section{Solving the cubic complex Ginzburg-Laundau equation by Homotopy analysis method}

\author{
S Naghshband ${ }^{1 *}$, M A Fariborzi Araghi ${ }^{1}$ \\ 1 Department of Mathematics, Central Tehran Branch, Islamic Azad University, Tehran, Iran
}

\section{Abstract}

Objectives: This paper obtains the series solution of the cubic complex Ginzburg-Laundau equation, by means of homotopy analysis method(HAM). Methods: In addition to the homotopy analysis method, homotopy perturbation and Adomian decomposition methods are applied to determine approximation solution of the cubic complex Ginzburg-Laundau equation and advantage of using HAM. Also a theorem is proved to guarantee the convergence of the HAM to solve this equation. Findings:Three examples are solved to illustrate the efficiency of the proposed method, this method is compared with other analytical approximate methods such as homotopy perturbation method (HPM)and Adomiam decomposition method(ADM) and it can be seen that these methods have the same results for this equation. Application: Homotopy analysis method as a reliable and valid scheme can be used to work out the cubic complex Ginzburg-Laundau equation which is nonlinear partial differential equation.

Keywords: Homotopy analysis method; Ginzburg-Laundau

\section{Introduction}

The Ginzburg-Laundau equation is one of the partial differential equations which occurs in chemical reactions, fluid mechanic, and many other sciences. The cGL is the general amplitude model which is describing the slow phase and amplitude modulations of a spatially distributed assembly of coupled oscillators close to its Hopf bifurcation ${ }^{(1)}$. The cubic cGL equation has been applied to investigate various applied issues such as chemical turbulence, Poiseuille flow, Taylor-Coutte flow, Rayleigh-Benard convection, reaction-diffusion systems, nonlinear optics, and hydrodynamical stability problems. It shows rich dynamics and has been a model for the transition to spatio-temporal chaos. The cGL can be considered 
as an usual form for a Hopf bifurcation in different types of spatially extended systems ${ }^{(2)}$.

We consider cubic complex Ginzburg-Laundau equation(cGL) as follows.

$$
\frac{\partial w}{\partial t}=(1+i a) \frac{\partial^{2} w}{\partial x^{2}}+R w-(1+i b)|w|^{2} w, w(x, 0)=f(x), i^{2}=-1
$$

where $a, b, R$ are real constants and $w=w(x, t)$ is a complex unknown function and $t$ is a nonnegative real quantity, also $x$ is real ${ }^{(3-7)}$. The complex field $w$ describes the modulations of the oscillator field $b$ and $R$ are two real control parameters. In point of fact, the amplitude $w$ explains slow modulations in space and time of the underlying bifurcating spatially periodical pattern ${ }^{(8)}$, also some other details about other types of this equation can be considered in ${ }^{(9-12)}$. Wazwaz studied this equation by using the separation of variables method in ${ }^{(13)}$. In this work, the HAM, HPM, ADM are considered in order to obtain the approximate solution of Equation (1). HAM is a strong analytical method to solve the nonlinear topics and was first introduced and applied ${ }^{(14)}$. Lately, this method has been well used to work out plenty types of problems in different branches of science and engineering ${ }^{(15-25)}$. Homotopy analysis method has an auxiliary parameter $h$ which gives us an easy approach to regulate the convergent region and the rate of convergence of the series solution. HAM mostly generates a very fast convergence of the solution series, usually just a few iterations attendant sufficient approximate solution, as well ${ }^{(24)}$. Likewise, a theorem will be proved which illustrates the convergence of HAM. Also HPM and ADM present satisfactory results ${ }^{(26,27)}$. Total explanation of the present paper is as follows: In section 2, some preliminaries are given, and in section 3 , the main idea of this paper is explained. In section 4 , the convergence theorem is proved, and finally in section 5 three examples are solved by all three methods, and $h$-curves are plotted to show the region of convergence.

\section{Preliminaries}

Let the following partial differential equation:

$$
N[w(x, t)]=0
$$

where $N$ is a nonlinear operator, $x$ and $t$ define the independent variables and $w$ is an unknown function. Via HAM, the zeroth-order deformation equation is:

$$
(1-q) L\left[\Phi(x, t, q)-w_{0}(x, t)\right]=q h H(x, t) N[\Phi(x, t, q)]
$$

Where $q \in[0,1]$ is the embedding parameter, $h \neq 0$ is an auxiliary parameter, $L$ is an auxiliary linear operator and $H(x, t)$ is an auxiliary function. $\Phi(x, t, q)$ is an unknown function and $w_{0}(x, t)$ is an initiative approximation of $w(x, t)$ It is obvious, if $q=0$ and $q=1$ then:

$$
\Phi(x, t, 0)=w_{0}(x, t), \Phi(x, t, 1)=w(x, t)
$$

respectively. Therefore, when $q$ increases from 0 to 1 , the solution $\Phi(x, t, q)$ varies from $w_{0}(x, t)$ to the exact solution $w(x, t)$. Via Taylor's theorem, it can be expanded $\Phi(x, t, q)$ in a power series of the embedding parameter $q$ as comes:

$$
\Phi(x, t, q)=w_{0}(x, t)+\sum_{m=1}^{\infty} w_{m}(x, t) q^{m}
$$


where

$$
w_{m}(x, t)=\left.\frac{1}{m !} \frac{\partial^{m} \Phi(x, t, q)}{\partial q^{m}}\right|_{q=0}
$$

Let the initiative approximation $w_{0}(x, t)$ the auxiliary linear operator $L$, the nonzero auxiliary parameter $h$ and the auxiliary function $H(x, t)$ be correctly selected so that the power series Equation (3) converges at $q=1$, then, it can be seen:

$$
w(x, t)=w_{0}(x, t)+\sum_{m=1}^{\infty} w_{m}(x, t)
$$

which must be the solution of the main nonlinear equation. Here, we consider the following set of vectors:

$$
\vec{w}_{n}=\left\{w_{0}(x, t), w_{1}(x, t), \ldots, w_{n}(x, t)\right\}
$$

By differentiating the zeroth order deformation Equation (2), $m$ times with regarding the embedding parameter $q$ and then putting $q=0$ and ultimately dividing by $m$ !. we will have the following $m$ th order deformation equation:

$$
L\left[w_{m}(x, t)-\chi_{m} w_{m-1}(x, t)\right]=h H(x, t) R_{m}\left(\vec{w}_{m-1}\right)
$$

where

$$
R_{m}\left(\vec{w}_{m-1}\right)=\left.\frac{1}{(m-1) !} \frac{\partial^{m-1} N[\Phi(x, t, q)]}{\partial q^{m-1}}\right|_{q-0}
$$

and

$$
\chi_{m}= \begin{cases}0 & m \leq 1 \\ 1 & m>1\end{cases}
$$

It shoud be mentioned that $w_{m}(x, t)$ for $m \geq 1$ is governed by the linear Equation (7) with linear boundary situations that arise from the main problem. In order to study more about the HAM, ${ }^{(28)}$ is suggested.

\section{Main Idea}

In this section, we apply the HAM, HPM and ADM to solve Equation (1) .

\subsection{Homotopy analysis method}

We consider Equation (1) as follows:

$$
\frac{\partial w}{\partial t}=(1+i a) \frac{\partial^{2} w}{\partial x^{2}}+R w-(1+i b) w^{2} \bar{w}, w(x, 0)=f(x), i^{2}=-1
$$

and

$$
L[\Phi(x, t, q)]=\frac{\partial \Phi(x, t, q)}{\partial t}, L(c)=0
$$


where $c$ is a real constant,

$$
N[\Phi(x, t, q)]=\frac{\partial \Phi(x, t, q)}{\partial t}-(1+i a) \frac{\partial^{2} \Phi(x, t, q)}{\partial x^{2}}-R \Phi(x, t, q)+(1+i b) \Phi^{2}(x, t, q) \bar{\Phi}(x, t, q)
$$

and $H(x, t)=1$. The zeroth-order deformation equation is:

$$
(1-q) L\left[\Phi(x, t, q)-w_{0}\right]=q h N[\Phi(x, t, q)]
$$

Also, the $m$ th-order deformation equation:

$$
L\left[w_{m}-\chi_{m} w_{m-1}\right]=h R_{m}\left(\omega \vec{w}_{m-1}\right)
$$

Where

$$
R_{m}\left(\vec{w}_{m-1}\right)=\frac{\partial w_{m-1}}{\partial t}-(1+i a) \frac{\partial^{2} w_{m-1}}{\partial x^{2}}-R w_{m-1}+(1+i b) \sum_{k=0}^{m-1} \sum_{j=0}^{k} w_{j} w_{k-j} \bar{w}_{m-1-k}
$$

So,

$$
w_{m}=\chi_{m} w_{m-1}+h \int_{0}^{t} R_{m}\left(\vec{w}_{m-1}\right) d t+c, m \geq 1
$$

\subsection{Homotopy perturbation method}

Consider Equation (10) as cubic complex Ginzburg-Laundau equation(cGL), to work out Equation (10) via homotopy perturbation method, we make the following homotopy

$$
(1-p)\left(\frac{\partial w}{\partial t}-\frac{\partial w_{0}}{\partial t}\right)+p\left(\frac{\partial w}{\partial t}-(1+a i) \frac{\partial^{2} w}{\partial x^{2}}-R w+(1+i b) w^{2} \bar{w}\right)=0
$$

or

$$
\frac{\partial w}{\partial t}-\frac{\partial w_{0}}{\partial t}=p\left(-\frac{\partial w_{0}}{\partial t}+(1+a i) \frac{\partial^{2} w}{\partial x^{2}}+R w-(1+i b) w^{2} \bar{w}\right)
$$

Suppose, the solution of Equation (10) is in the following form

$$
w=w_{0}+p w_{1}+p^{2} w_{2}+\ldots
$$

by putting Equation (18) into Equation (17), and equating the coefficients of the parts with the same powers of $p$, we get

$$
\begin{gathered}
p^{0}: \frac{\partial w_{0}}{\partial t}=\frac{\partial w_{0}}{\partial t} \\
p^{1}: \frac{\partial w_{1}}{\partial t}=-\frac{\partial w_{0}}{\partial t}+(1+a i) \frac{\partial^{2} w_{0}}{\partial x^{2}}+R w_{0}-(1+i b) w_{0}^{2} \bar{w}_{0}, w_{1}(x, 0)=0
\end{gathered}
$$




$$
\begin{gathered}
p^{2}: \frac{\partial w_{2}}{\partial t}=(1+a i) \frac{\partial^{2} w_{1}}{\partial x^{2}}+R w_{1}-(1+i b)\left(2 w_{0} \bar{w}_{\partial} w_{1}+\bar{w}_{1} w_{0}^{2}\right), w_{2}(x, 0)=0 \\
p^{j}: \frac{\partial w_{j}}{\partial t}=(1+i a) \frac{\partial^{2} w_{j-1}}{\partial x^{2}}+R w_{j-1}-(1+i b)\left(\sum_{i=0}^{j-1} \sum_{k=0}^{j} w_{k} w_{i-k} \bar{w}_{j-t-1}\right), w_{j}(x, 0)=0
\end{gathered}
$$

Obviously,

$$
w_{0}(x, t)=w_{0}(x, t)=f(x)
$$

and, by having these assumptions, we can write the following recursive relation

$$
\begin{gathered}
w_{1}(x, t)=\int_{0}^{t}\left(-\frac{\partial w_{0}}{\partial t}+(1+a i) \frac{\partial^{2} w_{0}}{\partial x^{2}}+R w_{0}-(1+i b) w_{0}^{2} \bar{w}_{0}\right) d t \\
w_{j}(x, t)=\int_{0}^{t}\left((1+a i) \frac{\partial^{2} w_{j-1}}{\partial x^{2}}+R w_{j-1}-(1+i b)\left(\sum_{i=0}^{j-1} \sum_{k=0}^{i} w_{k} w_{t-k} \bar{w}_{j-t-1} d t, j \geq 2\right.\right.
\end{gathered}
$$

The approximate solution of Equation (10) can be obtained by setting $p=1$, in Equation (18), that is,

$$
w=\lim _{p \rightarrow 1} w_{0}+p w_{1}+p^{2} w_{2}+\ldots=w_{0}+w_{1}+w_{2}+\ldots
$$

\subsection{Adomian decomposition method}

Consider Equation (10) and operator $L_{t}=\frac{\partial}{\partial t}$, applying the inverse operator $L_{t}^{(-1)}=\int_{0}^{t}() d$.$t to both sides$ of Equation (10), we have

$$
w(x, t)=w(x, 0)+\int_{0}^{t}\left((1+i a) \frac{\partial^{2} w}{\partial x^{2}}+R w-(1+i b) w^{2} \bar{w}\right) d t
$$

then

$$
w(x, t)=f(x)+\int_{0}^{t}\left((1+i a) \frac{\partial^{2} w}{\partial x^{2}}+R w-(1+i b) w^{2} \bar{w}\right) d t
$$

to solve Equation (10) by $\mathrm{ADM}$, as usual the series solution $w=\sum_{n=0}^{\infty} w_{n}$ is considered.then, the components $w_{n}$ can be determined recursively, now we consider $w^{2} \bar{w}=\sum_{n=0}^{\infty} A_{n}$, where $A_{n}\left(w_{0}, w_{1}, \ldots, w_{n}\right), \quad n \geq$ 0 are Adomian's polynomials which are computed by using this method, so we get

$$
\sum_{n=0}^{\infty} w_{n}=f(x)+\int_{0}^{t}\left[(1+i a) \frac{\partial^{2} \sum_{n=0}^{\infty} w_{n}}{\partial x^{2}}+R \sum_{n=0}^{\infty} w_{n}-(1+i b) \sum_{n=0}^{\infty} A_{n}\right] d t
$$


then, we obtain the following recursive relation:

$$
\begin{gathered}
w_{0}(x, t)=f(x) \\
w_{n+1}=\int_{0}^{t}\left((1+i a) \frac{\partial^{2} w_{n}}{\partial x^{2}}+R w_{n}-(1+i b) A_{n}\right) d t, n \geq 0
\end{gathered}
$$

where,

$$
\begin{gathered}
A_{0}=w_{0}^{2} \bar{w}_{0} \\
A_{1}=2 w_{0} w_{1} \bar{w}_{0}+w_{0}^{2} \bar{w}_{1} \\
A_{2}=2 w_{0} w_{2} \bar{w}_{0}+w_{1}^{2} \overline{w_{0}}+2 w_{0} w_{1} \bar{w}_{1}+w_{0}^{2} \overline{w_{2}} \\
A_{3}=2 w_{0} w_{3} \bar{w}_{0}+2 w_{1} w_{2} \bar{w}_{0}+2 w_{0} w_{2} \bar{w}_{1}+w_{1}^{2} \bar{w}_{1}+2 w_{0} w_{1} \bar{w}_{2}+w^{2} \bar{w}_{3}, \ldots
\end{gathered}
$$

\section{Convergence of the HAM}

In this section, we prove the convergence of the series solution obtained from the HAM to the exact solution of the Equation (10).

Theorem: If the series solution

$$
w(x, t)=w_{0}(x, t)+w_{1}(x, t)+\ldots
$$

obtained from the HAM is convergent, it converges to the exact solution of the Equation (10).

proof: Let the series

$$
\sum_{m=0}^{\infty} w_{m}(x, t)
$$

be convergent. Then we consider

$$
w(x, t)=\sum_{m=0}^{\infty} w_{m}(x, t)
$$

In this case, we can write,

$$
\lim _{m \rightarrow \infty} w_{m}(x, t)=0
$$

So

$$
\sum_{m-1}^{n}\left[w_{m}(x, t)-\chi_{m} w_{m-1}(x, t)\right]=w_{n}(x, t)
$$


assuming convergence of series solution, we have:

$$
\sum_{m=1}^{\infty}\left[w_{m}(x, t)-\chi_{m} w_{m-1}(x, t)\right]=\lim _{n \rightarrow \infty} w_{n}(x, t)=0
$$

then,

$$
\sum_{m-1}^{\infty} L\left[w_{m}(x, t)-\chi_{m} w_{m-1}(x, t)\right]=L\left(\sum_{m=1}^{\infty}\left(w_{m}(x, t)-\chi_{m} w_{m-1}(x, t)\right)\right)=0
$$

By applying the following statment

$$
L\left[w_{m}(x, t)-\chi_{m} w_{m-1}\right]=h H(x, t) R_{m}\left(\vec{w}_{m-1}\right)
$$

we get:

$$
\sum_{m-1}^{\infty} L\left[w_{m}(x, t)-\chi_{m} w_{m-1}\right]=h H(x, t) \sum_{m=1}^{\infty} R_{m}\left(\bar{w}_{m-1}\right)
$$

Moreover, since $h, H(x, t) \neq 0$,

$$
\sum_{m=1}^{\infty}\left[R_{m}\left(\bar{w}_{m-1}\right)\right]=0
$$

According to Equation (15), it can be seen:

$$
\begin{gathered}
\sum_{m=1}^{\infty}\left[R_{m}\left(\bar{w}_{m-1}\right)\right]=\sum_{m=1}^{\infty} \frac{\partial w_{m-1}}{\partial t}-(1+i a) \sum_{m=1}^{\infty} \frac{\partial^{2} w_{m-1}}{\partial x^{2}}-R \sum_{m=1}^{\infty} w_{m-1}+ \\
(1+i b) \sum_{m=1}^{\infty} \sum_{k=0}^{m-1} \sum_{j=0}^{k} w_{j} w_{k-j} \bar{w}_{m-1-k}
\end{gathered}
$$

Therefore,

$$
\begin{gathered}
\sum_{m=1}^{\infty}\left[R_{m}\left(\bar{w}_{m-1}\right)\right]=\sum_{m=1}^{\infty} \frac{\partial w_{m-1}}{\partial t}-(1+i a) \sum_{m=1}^{\infty} \frac{\partial^{2} w_{m-1}}{\partial x^{2}}-R \sum_{m=1}^{\infty} w_{m-1}+ \\
(1+i b) \sum_{k=0}^{\infty} \sum_{m-k+1}^{\infty} \sum_{j=0}^{k} w_{j} w_{k-j} \bar{w}_{m-k-1}
\end{gathered}
$$

then

$$
\begin{aligned}
& \sum_{m-1}^{\infty}\left[R_{m}\left(\bar{w}_{m-1}\right)\right]=\sum_{m=1}^{\infty} \frac{\partial w_{m-1}}{\partial t}-(1+i a) \sum_{m=1}^{\infty} \frac{\partial^{2} w_{m-1}}{\partial x^{2}}-R \sum_{m-1}^{\infty} w_{m-1}+ \\
&(1+i b) \sum_{j=0}^{\infty} \sum_{k=1}^{\infty} \sum_{m=1}^{\infty} w_{j} w_{k-j} \bar{w}_{m-1}
\end{aligned}
$$

so, we obtain

$$
\begin{aligned}
\sum_{m=1}^{\infty}\left[R_{m}\left(\bar{w}_{m-1}\right)\right]= & \sum_{m=1}^{\infty} \frac{\partial w_{m-1}}{\partial t}-(1+i a) \sum_{m=1}^{\infty} \frac{\partial^{2} w_{m-1}}{\partial x^{2}}-R \sum_{m=1}^{\infty} w_{m-1}+ \\
& (1+i b) \sum_{j=0}^{\infty} \sum_{k=0}^{\infty} \sum_{m-1}^{\infty} w_{j} w_{k} \bar{w}_{m-1}
\end{aligned}
$$

then, it is found that

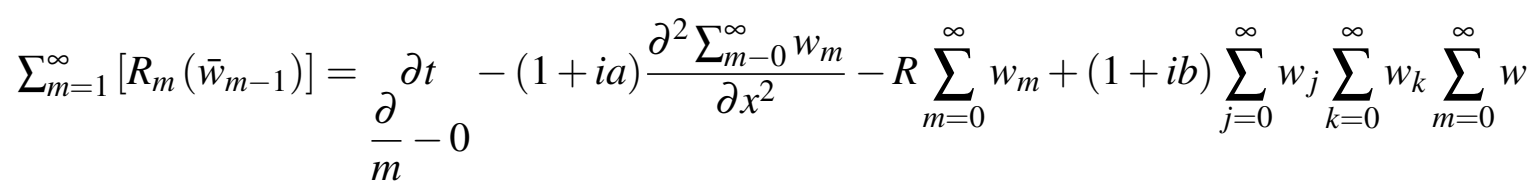

From Equation (29) and Equation (30) and the relation

$$
\sum_{m-0}^{\infty} \bar{w}=\sum_{m=0}^{\infty} w_{m}
$$

we conclude that

$$
w(x, t)=\sum_{m=0}^{\infty} w_{m}(x, t)
$$

is the exact solution of Equation (10). 


\section{Sample Examples}

In this section, we solve three cubic complex Ginzburg-Laundau equations via the HAM and the results are compared with the ADM and the HPM. Also, the region of convergence are shown in the HAM by plotting the $h$-curves. The programs have been provided and the figures have been plotted by Matlab package.

Example 1: Consider the following cGL equation:

$$
w_{t}=(1-3 / 2 i) w_{x x}+2 w-(1+i / 2)|w|^{2} w, w(x, 0)=e^{i x}, i^{2}=-1
$$

We solve the equation by the HAM, using Equation (16), we get:

$$
\begin{gathered}
w_{0}(x, t)=e^{i x} \\
w_{1}(x, t)=-h t i e^{x i} \\
w_{2}(x, t)=-\left(h t e^{x i}(2 h i+h t+2 i)\right) / 2 \\
w_{3}(x, i)=-\left(h t e^{x t}\left(-h^{2} t^{2} i+6 h^{2} t+6 h^{2} i+6 h t+12 h i+6 i\right)\right) / 6, \ldots
\end{gathered}
$$

When $h=-1$, we can write

$$
\begin{gathered}
w_{0}(x, i)=e^{k} \\
w_{1}(x, t)=i t e^{k} \\
w_{2}(x, t)=-\left(t^{2} e^{x i}\right) / 2=\frac{(i t)^{2}}{2 !} e^{\hbar} \\
w_{3}(x, t)=\frac{-\left(i t^{3} e^{i x}\right)}{6}=\frac{(i t)^{3}}{3 !} e^{i}, \ldots . .
\end{gathered}
$$

so, we can easily see

$$
\begin{array}{r}
w(x, t)=w_{0}(x, t)+w_{1}(x, t)+w_{2}(x, t)+w_{3}(x, t)+\ldots= \\
e^{t \pi}+i t e^{t}+\frac{(i t)^{2}}{2 !} e^{t x}+\frac{(i t)^{3}}{3 !} e^{t t}+\ldots=e^{i(x+t)}
\end{array}
$$

that is the exact solution of the equation.

Table 1 shows the errors of HAM at $x=-2$ with different $\mathrm{t}$ when $n=12$ and $h=-1$ 
Table 1. The errors of the HAM at $x=-2$ when $n=12$ and $h=-1$

\begin{tabular}{lllllll}
\hline $\mathrm{t}$ & 0.2 & 0.4 & 0.6 & 0.8 & 1 \\
\hline Error & $2.2204 \mathrm{e}-016$ & $9.9093 \mathrm{e}-016$ & $2.0959 \mathrm{e}-013$ & $8.8161 \mathrm{e}-012$ & $1.6024 \mathrm{e}-010$ \\
\hline
\end{tabular}

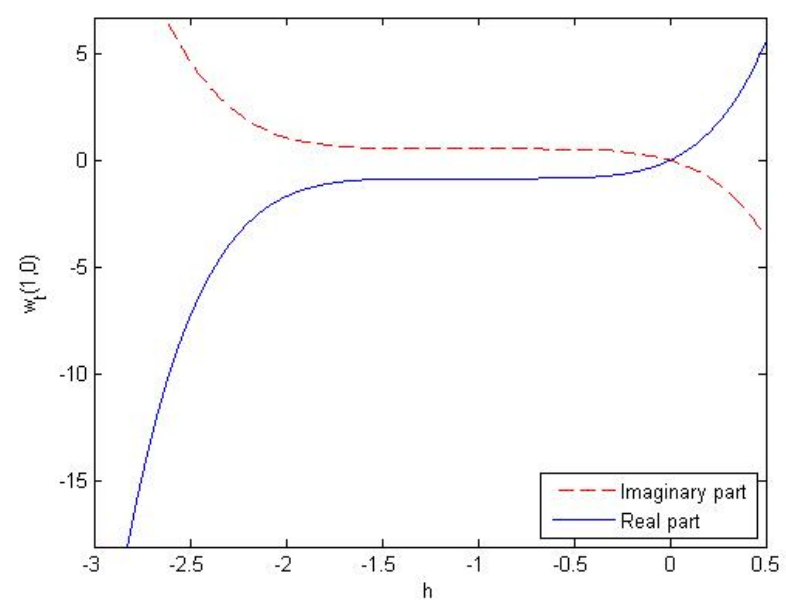

Fig 1. The $h$-curve of 5-approximation $(n=5)$ of example1 when $x=1$ and $t=0$

Table 2. The errors of the HAM at the point $(2,1)$

\begin{tabular}{lll}
\hline $\mathrm{n}$ & Approximation at by HAM $(h=-1)$ & Error \\
\hline 2 & $-1.117370845099253 \mathrm{e}+000+3.850187686569845 \mathrm{e}-002 \mathrm{i}$ & $1.635717717580491 \mathrm{e}-001$ \\
4 & $-9.831607254844369 \mathrm{e}-001+1.457470757412923 \mathrm{e}-001 \mathrm{i}$ & $8.251233356899829 \mathrm{e}-003$ \\
6 & $-9.901602223238910 \mathrm{e}-001+1.410162723439193 \mathrm{e}-001 \mathrm{i}$ & $1.972131260047780 \mathrm{e}-004$ \\
8 & $-9.899901272698717 \mathrm{e}-001+1.411213931802091 \mathrm{e}-001 \mathrm{i}$ & $2.744500998182562 \mathrm{e}-006$ \\
10 & $-9.899925183709056 \mathrm{e}-001+1.411199958130925 \mathrm{e}-001 \mathrm{i}$ & $2.497871950008141 \mathrm{e}-008$ \\
12 & $-9.899924964598675 \mathrm{e}-001+1.411200081367663 \mathrm{e}-001 \mathrm{i}$ & $1.602361278494555 \mathrm{e}-010$ \\
14 & $-9.899924966011185 \mathrm{e}-001+1.411200080595068 \mathrm{e}-001 \mathrm{i}$ & $7.635667678970700 \mathrm{e}-013$ \\
16 & $-9.899924966004430 \mathrm{e}-001+1.411200080598685 \mathrm{e}-001 \mathrm{i}$ & $2.769027319221402 \mathrm{e}-015$ \\
\hline
\end{tabular}

Figure 1 shows, the region of convergence of the example 1 at the point $(1,0)$, which is $-1.5<h<0$

Table 2 shows the convergence of the HAM at the point $(2,1)$ and $n=2,4,6,8,10,12,14,16$ and error is calculated by $\left|\sum_{i=0}^{n} w_{i}-w\right|$

Now, we solve the equation by HPM to compare the results. By using Equation (19) and Equation (20), we obtain

$$
\begin{gathered}
w_{0}(x, t)=e^{x} \\
w_{1}(x, t)=t i e^{i x} \\
w_{2}(x, t)=-\left(t^{2} e^{i x}\right) / 2=\frac{(i t)^{2}}{2 !} e^{i x}
\end{gathered}
$$




$$
w_{3}(x, t)=-\left(t^{3} e^{x i} i\right) / 6=\frac{(i t)^{3}}{3 !} e^{i x}, \ldots
$$

so, we get

$$
\begin{gathered}
w(x, t)=w_{0}(x, t)+w_{1}(x, t)+w_{2}(x, t)+w_{3}(x, t)+\ldots= \\
e^{i x}+i t e^{i x}+\frac{(i t)^{2}}{2 !} e^{i x}+\frac{(i t)^{3}}{3 !} e^{i x}+\ldots=e^{i(x+t)}
\end{gathered}
$$

which is the same results of HAM when $(h=-1)$. The results of the ADM by using Equation (22) are as follows:

$$
\begin{gathered}
w_{0}(x, t)=e^{i x} \\
w_{1}(x, t)=t i e^{i x} \\
w_{2}(x, t)=-\left(t^{2} e^{i x}\right) / 2=\frac{(i t)^{2}}{2 !} e^{i x} \\
w_{3}(x, t)=-\left(t^{3} e^{x i} i\right) / 6=\frac{(i t)^{3}}{3 !} e^{i x}, \ldots
\end{gathered}
$$

therefore, we can write

$$
\begin{gathered}
w(x, t)=w_{0}(x, t)+w_{1}(x, t)+w_{2}(x, t)+w_{3}(x, t)+\ldots= \\
e^{i x}+i t e^{i x}+\frac{(i t)^{2}}{2 !} e^{i x}+\frac{(i t)^{3}}{3 !} e^{i x}+\ldots=e^{i(x+t)}
\end{gathered}
$$

which is the same results of HPM and HAM when $(h=-1)$. It can be seen that all three methods are able to produce the similar results, via the HAM, we can avoid of difficulties in calculation of Adomian's polynomials specially for larger values of $n$, and computation of powers of $p$ by the HPM.

Example 2: Consider the following cGL:

$$
w_{t}=(1-9 i) w_{x x}+10 / 9 w-(1-i)|w|^{2} w, w(x, 0)=e^{-i x / 3}, i^{2}=-1
$$

By using Equation (16), namely HAM, it can be seen:

$$
\begin{gathered}
w_{0}(x, t)=e^{-i x / 3} \\
w_{1}(x, t)=-2 h t\left(1 / e^{(i x) / 3)} i\right.
\end{gathered}
$$




$$
\begin{gathered}
w_{2}(x, t)=-2 h t\left(1 / e^{(i x) / 3)}(h i+h t+i)\right. \\
w_{3}(x, t)=-\left(4 h t\left(1 / e^{(x x) / 3)}\left(-h^{2} t^{2} i+3 h^{2} t+\left(3 h^{2} i\right) / 2+3 h t+3 h i+(3 i) / 2\right)\right) / 3, \ldots\right.
\end{gathered}
$$

When $(h=-1)$, we can see

$$
\begin{gathered}
w_{0}(x, t)=e^{-i x / 3} \\
w_{1}(x, t)=2 t\left(1 / e^{(i x) / 3} i=2 t i e^{-i x / 3}\right. \\
w_{2}(x, t)=-2 t^{2}\left(1 / e^{i x / 3}\right)=\frac{(2 t i)^{2} e^{-i x / 3}}{2 !} \\
w_{3}(x, t)=-\left(4 t^{3}\left(1 / e^{i x / 3}\right) i\right) / 3=\frac{(2 t i)^{3} e^{-i x / 3}}{3 !}, \ldots
\end{gathered}
$$

so,

$$
\begin{gathered}
w(x, t)=w_{0}(x, t)+w_{1}(x, t)+w_{2}(x, t)+w_{3}(x, t)+\ldots= \\
e^{-i x / 3}+2 t i e^{-i x / 3}+\frac{(2 t i)^{2}}{2 !} e^{-i x / 3}+\frac{(2 t i)^{3}}{3 !} e^{-i x / 3}+\ldots=e^{2 t i-i x / 3}
\end{gathered}
$$

which is the accurate solution of the equation.

Figure 2 presents the convergence region of the example 2 at the point $(-1,0)$.

Table 3 shows the errors of HAM $(\mathrm{h}=-1)$ at $\mathrm{x}=1$ with different values of $\mathrm{t}$, when $\mathrm{n}=18$.

Table 3. The errors of HAM $(\mathrm{h}=-1)$ at $\mathrm{x}=1$, when $\mathrm{n}=18$

\begin{tabular}{llllll}
\hline $\mathrm{t}$ & 0.2 & 0.4 & 0.6 & 0.8 & 1 \\
\hline Error & $1.1189 \mathrm{e}-016$ & $3.1402 \mathrm{e}-016$ & $1.5701 \mathrm{e}-016$ & $6.1733 \mathrm{e}-014$ & $4.2904 \mathrm{e}-012$ \\
\hline
\end{tabular}

Figure 3 shows the real part of the approximate solution for $n=6$ and the real part of exact solution, respectively. Also, Figure 4 compares the imaginary part of the approximate solution for $n=6$ and the exact solution respectively.

Table 4 shows the convergence of this method at the point $(1,0.6)$ and for $n=3,6,9,12,15,18$ and the error is $\left|\sum_{i=0}^{n} w_{i}-w\right|$

Table 5 shows the errors, at the point $(1,0.6)$, for different values of $h$.

Applying HPM, namely Equation (19) and Equation (20), we get

$$
w_{0}(x, t)=e^{-i x / 3}
$$




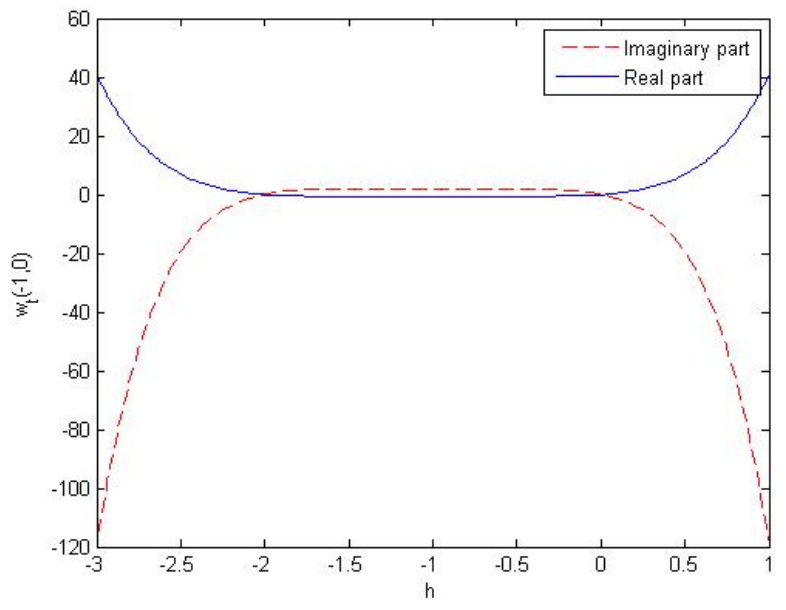

Fig 2. The -curve of 6 -approximation $(n=2)$ of example 2 when $x=-1$ and $t=0$
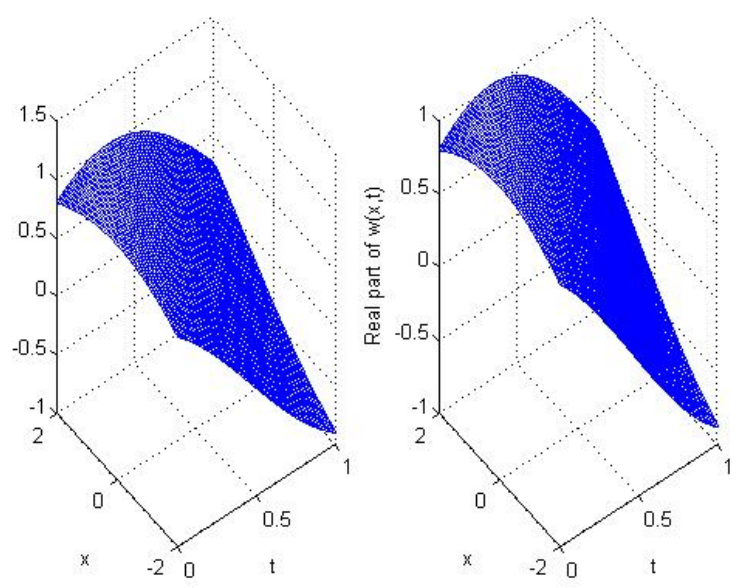

Fig 3. Real parts of the approximate(left) and the exact solutions of example 2 when $n=6$ and $h=-1$

Table 4. The errors of the HAM at the point $(1,0.6)$.

\begin{tabular}{lll}
\hline $\mathrm{n}$ & Approximation at $(1,0.6)$ via HAM $(\mathrm{h}=-1)$ & Error \\
\hline 3 & $5.629895084462173 \mathrm{e}-001+7.701862199361181 \mathrm{e}-001 \mathrm{i}$ & $8.476063171568474 \mathrm{e}-002$ \\
6 & $6.474995723928192 \mathrm{e}-001+7.628681672182660 \mathrm{e}-001 \mathrm{i}$ & $7.047727373065257 \mathrm{e}-004$ \\
9 & $6.473723785312548 \mathrm{e}-001+7.621748949527030 \mathrm{e}-001 \mathrm{i}$ & $1.697855943744531 \mathrm{e}-006$ \\
12 & $6.473707228589358 \mathrm{e}-001+7.621752712533816 \mathrm{e}-001 \mathrm{i}$ & $1.712756669817200 \mathrm{e}-009$ \\
15 & $6.473707232781010 \mathrm{e}-001+7.621752729140687 \mathrm{e}-001 \mathrm{i}$ & $8.816695142989886 \mathrm{e}-013$ \\
18 & $6.473707232789525 \mathrm{e}-001+7.621752729138399 \mathrm{e}-001 \mathrm{i}$ & $1.570092458683775 \mathrm{e}-016$ \\
\hline
\end{tabular}



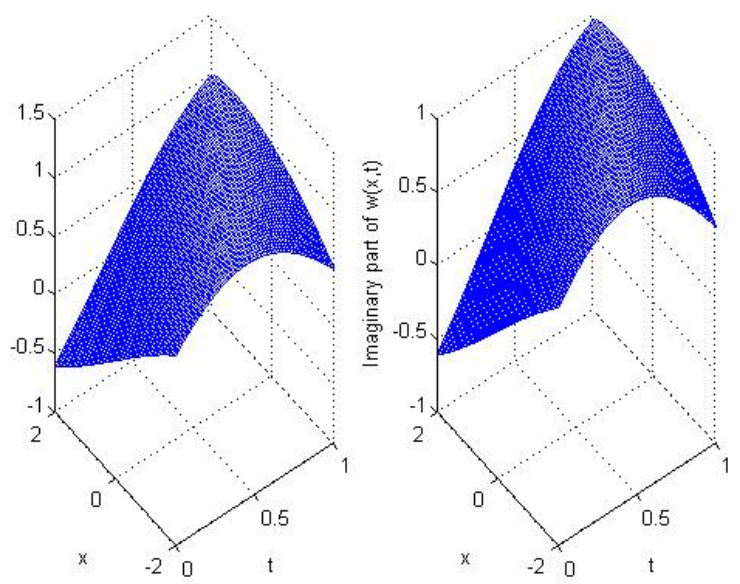

Fig 4. Imaginary parts of the approximate(left) and the exact solutions of example 2 when $\mathrm{n}=6$ and $\mathrm{h}=-1$

Table 5. The errors of the HAM, at the point $(1,0.6)$

\begin{tabular}{lllll}
\hline $\mathrm{h}$ & -1.25 & -1 & -0.75 & -0.5 \\
\hline 3 & $2.8584 \mathrm{e}-001$ & $8.4761 \mathrm{e}-002$ & $1.3122 \mathrm{e}-001$ & $2.8949 \mathrm{e}-001$ \\
6 & $3.0911 \mathrm{e}-002$ & $7.0477 \mathrm{e}-004$ & $8.1473 \mathrm{e}-003$ & $6.7683 \mathrm{e}-002$ \\
9 & $2.3592 \mathrm{e}-003$ & $1.6979 \mathrm{e}-006$ & $4.0043 \mathrm{e}-004$ & $1.4635 \mathrm{e}-002$ \\
12 & $1.4666 \mathrm{e}-004$ & $1.7128 \mathrm{e}-009$ & $1.7133 \mathrm{e}-005$ & $3.0039 \mathrm{e}-003$ \\
15 & $7.9268 \mathrm{e}-006$ & $8.8167 \mathrm{e}-013$ & $6.6626 \mathrm{e}-007$ & $5.9337 \mathrm{e}-004$ \\
\hline
\end{tabular}

$$
\begin{gathered}
w_{1}(x, t)=2 t\left(1 / e^{(i x) / 33} i=2 t i e^{-i x / 3}\right. \\
w_{2}(x, t)=-2 t^{2}\left(1 / e^{i x / 3}\right)=\frac{(2 t i)^{2} e^{-i x / 3}}{2 !} \\
w_{3}(x, t)=-\left(4 t^{3}\left(1 / e^{i x / 3}\right) i\right) / 3=\frac{(2 t i)^{3} e^{-i x / 3}}{3 !}, \ldots
\end{gathered}
$$

So, we have

$$
\begin{gathered}
w(x, t)=w_{0}(x, t)+w_{1}(x, t)+w_{2}(x, t)+w_{3}(x, t)+\ldots= \\
e^{-i x / 3}+2 t i e^{-i x / 3}+\frac{(2 t i)^{2}}{2 !} e^{-i x / 3}+\frac{(2 t i)^{3}}{3 !} e^{-i x / 3}+\ldots=e^{2 t i-i x / 3}
\end{gathered}
$$

which is the same results of HAM when $\mathrm{h}=-1$. Using Equation (22), the results of the ADM are as follows:

$$
\begin{gathered}
w_{0}(x, t)=e^{-i x / 3} \\
w_{1}(x, t)=2 t\left(1 / e^{(i x) / 3} i\right)=2 t i e^{-i x / 3}
\end{gathered}
$$




$$
\begin{gathered}
w_{2}(x, t)=-2 t^{2}\left(1 / e^{i x / 3}\right)=\frac{(2 t i)^{2} e^{-i x / 3}}{2 !} \\
w_{3}(x, t)=-\left(4 t^{3}\left(1 / e^{i x / 3}\right) i\right) / 3=\frac{(2 t i)^{3} e^{-i x / 3}}{3 !}, . .
\end{gathered}
$$

therefore, we can write

$$
\begin{gathered}
w(x, t)=w_{0}(x, t)+w_{1}(x, t)+w_{2}(x, t)+w_{3}(x, t)+\ldots= \\
e^{-i x / 3}+2 t i e^{-i x / 3}+\frac{(2 t i)^{2}}{2 !} e^{-i x / 3}+\frac{(2 t i)^{3}}{3 !} e^{-i x / 3}+\ldots=e^{2 t i-i x / 3}
\end{gathered}
$$

Example 3: consider the following PDE:

$$
i u_{t}+u_{x x}+2|u|^{2} u-u+i u=0, u(x, 0)=e^{i x} 30
$$

Applying the HAM for this equation, we get

$$
\begin{gathered}
u_{0}(x, t)=e^{i x} \\
u_{1}(x, t)=h t e^{i x} \\
u_{2}(x, t)=\left(h t e^{i x}(2 h+2+h t(1-4 i))\right) / 2 \\
u_{3}(x, t)=\left(h t e^{i} x\left(h^{2} t^{2}(1-20 i)+h^{2} t(6-24 i)+6 h^{2}+h t(6-24 i)+12 h+6\right)\right) / 6 \\
u_{4}(x, t)=\left(h t e ^ { x i } \left(h^{3} t^{3}(-72 i-47)+h^{3} t^{2}(12-240 i)+h^{3} t(36-144 i)+24 h^{3}+h^{2} t^{2}(12-240 i)+\right.\right. \\
\left.\left.h^{2} t(72-288 i)+72 h^{2}+h t(36-144 i)+72 h+24\right)\right) / 24 \\
u_{5}(x, t)=\left(h t e ^ { x i } \left(h^{4} t^{4}(-232 i-559)+h^{4} t^{3}(-1440 i-940)+\mid h^{4} t^{2}(120-2400 i)+h^{4} t(240-960 i)+\right.\right. \\
120 h^{4}+h^{3} t^{3}(-1440 i-940)+h^{3} t^{2}(240-4800 i)+h^{3} t(720-2880 i)+480 h^{3}+ \\
\left.\left.h^{2} t^{2}(120-2400 i)+h^{2} t(720-2880 i)+720 h^{2}+h t(240-960 i)+480 h+120\right)\right) / 120
\end{gathered}
$$

Setting $\mathrm{h}=-1$, we obtain

$$
\begin{gathered}
u_{0}(x, t)=e^{i x} \\
u_{1}(x, t)=-t e^{i x}
\end{gathered}
$$




$$
\begin{gathered}
u_{2}(x, t)=t^{2} e^{x i}(1 / 2-2 i) \\
u_{3}(x, t)=t^{3} e^{x i}((10 i) / 3-1 / 6) \\
u_{4}(x, t)=t^{4} e^{x i}(-3 i-47 / 24) \\
u_{5}(x, t)=t^{5} e^{x i}((29 i) / 15+559 / 120)
\end{gathered}
$$

so with the help of these computations, we have

$$
\begin{gathered}
u(x, t)=u_{0}(x, t)+u_{1}(x, t)+u_{2}(x, t)+u_{3}(x, t)+\ldots= \\
e^{i x}-t e^{i x}+t^{2} e^{x i}(1 / 2-2 i)+t^{3} e^{x i}((10 i) / 3-1 / 6)+ \\
t^{4} e^{x i}(-3 i-47 / 24)+t^{5} e^{x i}((29 i) / 15+559 / 120)+\ldots= \\
\sum_{n=0}^{\infty} \frac{(-t)^{n}}{n !} e^{i x}+i e^{i x}\left(-2 t^{2}+10 / 3 t^{3}-3 t^{4}+29 / 15 t^{5}-\ldots\right)+e^{i x}\left(-2 t^{4}+4 t^{5}-\ldots\right)
\end{gathered}
$$

Which is the same series solution that has been calculated by HPM and $\operatorname{ADM}^{(29)}$. According to the hcurve at any point and by changing $h$. it is possible to obtain other approximation solution. Also, Figure 5 shows the region of convergence of the example 3 , when $x=1$ and $t=0$.

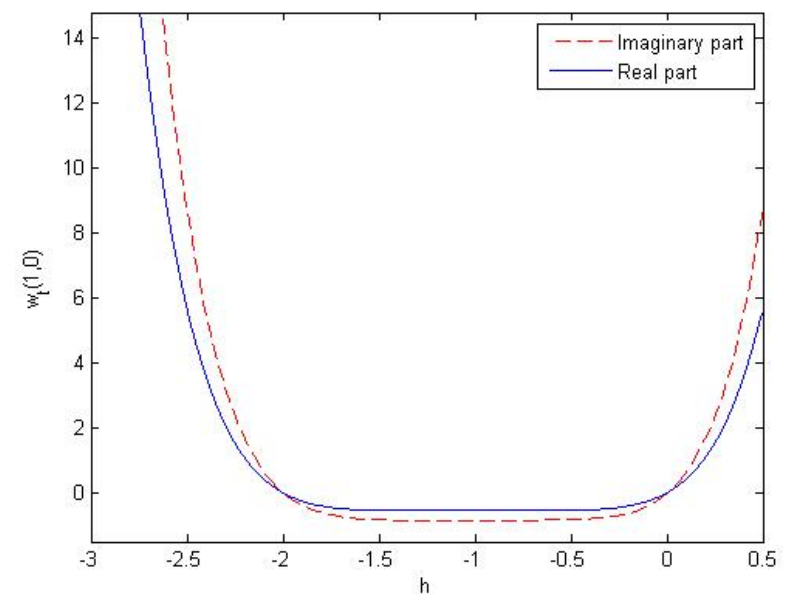

Fig 5. The h-curve of 6 -approximation $(n=6)$ of example 3 when $x=1$ and $t=0$. 


\section{Conclusion}

This paper used, the homotopy analysis method, homotopy perturbation method, and Adomian decomposition method to solve the cubic complex Ginzburg-Laundau equation and a theorem of convergence of the HAM was proved. Also, three examples were solved and the h-curves of the examples were drawn and some numerical results were presented to show the importance and applicability of the HAM, likewise one can observe that all three methods produce the similar results, however the HAM provides a situation that one can avoid of difficulties in calculation of Adomian's polynomials specially for larger values of $n$, and computation of powers of $\mathrm{p}$ by the HPM, also if necessary, by different values of $h$, region of convergence can be controlled via the HAM. Furthermore, the ADM and HPM are a specific case of the HAM when $\mathrm{h}=-1$. Consequently, the HAM can be used to work out the cGL as a reliable and valid scheme.

\section{Acknowledgement}

The authors would like to thank the Islamic Azad university, Central Tehran branch, and West Tehran branch, for their supports during this investigation.

\section{References}

1) Eguiluz V, Hernandez-Garcia E and Piro O. Boundary effects in the complex Ginzburg- Landau equation. International Journal of Bifurcation and Chaos in Applied Sciences and Engineering. 1999;9(11):2209-2214.

2) Wazwaz A. Partial Differential Equations and Solitary Waves Theory: Families of Camassa-Holm and Schrodinger Equations. In: and others, editor. Nonlinear physical science . 2009,. p. 693-694.

3) Charbonneau G. De l'hysérie masculine : Comprendre l'hysérie par son expression masculine. In: PSN. vol. 4. Vancouver, B.C. Canada. Springer Science and Business Media LLC . 2006,. p. 6-10. Available from: https://dx.doi.org/10.1007/ bf03028393.

4) Ginibre J and Velo G. The Cauchy Problem in Local Spaces for the Complex Ginzburg-Landau EquationgII. Contraction Methods. Communications in Mathematical Physics. 1997;187(1):45-79. Available from: https://dx.doi.org/10.1007/ s002200050129.

5) Gao $\mathrm{H}$ and Wang X. On the global existence and small dispersion limit for a class of complex Ginzburg-Landau equations. Mathematical Methods in the Applied Sciences. 2009;32:1396-1414.

6) Saarloos WV. The Complex Ginzburg-Landau equation for beginners. vol. 9506. Leiden The Netherlands . 1994,.

7) Yang Y and Gao H. Continuous dependence on modelling for a complex Ginzburg Landau equation with complex coefficients. Mathematical Methods in the Applied Sciences. 2004;27:1567-1578.

8) Marcq P, Chate $\mathrm{H}$ and Conte R. Exact solutions of the one-dimensional quintic complex Ginzburg-Landau equation. Physica D: Nonlinear Phenomena. 1994;73(4):305-317. Available from: https://dx.doi.org/10.1016/0167-2789(94)90102-3.

9) Aranson IS and Kramer L . 2001,.

10) Nasibov S. On a class of non-linear evolution Ginzburg-Landau-Schrodinger type equation. Applied and computational mathematics. 2004;3(2):142-151.

11) Sherratt JA, Smith MJ and Rademacher JDM. Patterns of Sources and Sinks in the Complex Ginzburg-Landau Equation with Zero Linear Dispersion. SIAM Journal on Applied Dynamical Systems. 2010;9(3):883-918. Available from: https: //dx.doi.org/10.1137/090780961.

12) Kshirsagar SW, Patil NR and Samant SD. Preparation of Mg-Al hydrotalcite from the effluent of Friedel-Crafts reaction and its application as a catalyst in Knoevenagel reaction. Green Chemistry Letters and Reviews. 2010;3(4):335-339. Available from: https://dx.doi.org/10.1080/17518253.2010.491094.

13) Wazwaz A . 2009,.

14) Liao SJ . 1992,.

15) Abbasbandy S. The application of homotopy analysis method to solve a generalized Hirota-Satsuma coupled KdV equation. Physics Letters A. 2007;361(6):478-483. Available from: https://dx.doi.org/10.1016/j.physleta.2006.09.105.

16) Abbasbandy S. The application of homotopy analysis method to nonlinear equations arising in heat transfer. Physics Letters A. 2006;360(1):109-113. Available from: https://dx.doi.org/10.1016/j.physleta.2006.07.065. 
17) Alizadeh-Pahlavan A, Aliakbar V, Vakili-Farahani F and Sadeghy K. MHD flows of UCM fluids above porous stretching sheets using two-auxiliary-parameter homotopy analysis method. Communications in Nonlinear Science and Numerical Simulation. 2009;14(2):473-488. Available from: https://dx.doi.org/10.1016/j.cnsns.2007.09.011.

18) Alomari AK, Noorani MSM and Nazar R. Explicit series solutions of some linear and nonlinear Schrodinger equations via the homotopy analysis method. Communications in Nonlinear Science and Numerical Simulation. 2009;14:1196-1207. Available from: https://dx.doi.org/10.1016/j.cnsns.2008.01.008.

19) Alomari AK, Noorani MSM and Nazar R. Comparison between the homotopy analysis method and homotopy perturbation method to solve coupled Schrodinger-KdV equation. Journal of Applied Mathematics and Computing. 2009;31(1-2):1-12. Available from: https://dx.doi.org/10.1007/s12190-008-0187-4.

20) Araghi MAF and Naghshband S. On convergence of homotopy analysis method to solve the Schrodinger equation with a power law nonlinearity. International Journal of Industrial Mathematics. 2013;5(4):367-374.

21) Sajid M, Hayat T and Asghar S. Comparison between the HAM and HPM solutions of thin film flows of non-Newtonian fluids on a moving belt. Nonlinear Dynamics. 2007;50(1-2):27-35. Available from: https://dx.doi.org/10.1007/s11071-0069140-y.

22) Fallahzadeh A and Araghi MAF. Dynamical Control of Accuracy using the Stochastic Arithmetic to Estimate the Solution of the Fuzzy Differential Equations via Homotopy Analysis Method. Indian Journal of Science and Technology. 2015;8(34). Available from: https://dx.doi.org/10.17485/ijst/2015/v8i34/50621.

23) Naghshband S and Araghi MAF. Approximation solution of the complex modified Korteweg-de Vries equation via the homotopy analysis method. Communications on Advanced Computational Science with Applications. 2017;2017(1):12-23. Available from: https://dx.doi.org/10.5899/2017/cacsa-00066.

24) Naghshband S, Araghi F and A M. Solving generalized quintic complex Ginzburg-Landau equation by homotopy analysis method. Ain Shams Engineering Journal. 2016;

25) Araghi MAF and Fallahzadeh A. On the convergevce of the homotopy analysis method for solving the Schrodinger equation. Journal of Basic and Applied Scientific Research. 2012;(2):6076-6083.

26) Biazar J, Ansari R, Hosseini K and Gholamian P . 2008,.

27) Wazwaz A. A new algorithm for calculating adomian polynomials for nonlinear operators. Applied mathematics and computation. 2000;111:53-69.

28) Liao SJ. Beyond perturbation:Introduction to the homotopy analysis method. Boca Raton. Chapman and Hall/CRC Press .2003,.

29) Biazar J, Partovi M and Ayati Z. Approximating Solutions for Ginzburg- Landau Equation by HPM and ADM. Applications and Applied Mathematics. 2010;p. 1672-1681. 